\title{
The Linguistic Analysis of Sound Ambiguity and Meaning Ambiguity
}

\author{
Congying Liu
}

\author{
Handan College Handan, Hebei Province, China
}

\begin{abstract}
This thesis will talk about ambiguity by classifying it into sound ambiguity and meaning ambiguity. In the first part, the author will discuss the theories in ambiguity namely the Q-and R-principle and IC-analysis which are the most basic and important in the analysis of ambiguity. In the following part, the author will analyze the sound ambiguity and meaning ambiguity in detail. Polysemy and homonymy are the main causes of sound ambiguity. Culture and context are the main causes of meaning ambiguity. And in the last part the author will discuss the advantage and disadvantage of ambiguity. Ambiguity can be seen as a sword that has two blades. The positive side should be studied to make the language becomes more interesting and vivid. The negative side should be avoided to make the writing or speech more accurate.
\end{abstract}

Index Terms: Ambiguity; sound meaning; context

(C) 2012 Published by MECS Publisher. Selection and/or peer review under responsibility of the International Conference on E-Business System and Education Technology

\section{Introduction}

In the West, ambiguity can be traced back to the ancient Greek philosophy. Plato studies ambiguity in philosophy. He indicates that ambiguity mostly lead to sophism and false inference. One of his pupils Aristotle makes a generally study of ambiguity. He says that ambiguity is a sentence with two or more propositions. Logicians study ambiguity from predicate logic, modal logic and prepositional logic. They believe that it is necessary to eliminate ambiguity and indeterminacy. At first, linguists do not pay much attention to ambiguity. They think it is the task of philosophers and logicians. However, Ferdinand de Saussure, father of modern linguistics, points out for the first time that the study of linguistic description must explain language phenomenon, ambiguity exists commonly in natural languages and it cannot be avoid.

\section{The Linguistic Theories in Ambiguity}

In Oxford Advanced Learner's English and Chinese Dictionary, ambiguity means the state of having more than one possible meaning. In Longman dictionary of language teaching and applied linguistics (third edition): A

* Corresponding author.

E-mail address: hdxylcy@163.com 
word, phrase, or sentence which has more than one meaning is said to be ambiguity. However, it is common for one word, phrase, or sentence to have more than one meaning in languages. There are good examples in both English and Chinese. In order to have a more complete picture about the topic the writer has examined, the author consult a lot of theories about this study. Ambiguity is a very interesting topic. The writer decides to use the Q-and R-principle and IC-analysis method to analyze the ambiguity on sound and meaning.

It is supposed that the interlocutors involved in conversation are generally cooperating with each other. On this study, Grice composes his co-operative principle, which includes four maxims: (1) Quantity Maxim: 1) Make your contribution as informative as is required (for the current purposes of the exchange). 2) Do not make your contribution more informative than is required. (2) Quality Maxim: Try to make your contribution one that is true, specifically. 1) Do not say what you believe to be false. 2) Do not say that for which you lack adequate evidence. (3)Relation Maxim: Be relevant. (4) Manner Maxim: Be perspicuous, and specifically: 1) Avoid obscurity of expression. 2) Avoid ambiguity. 3) Be brief. 4) Be orderly1.

Grice's CP specifies what participants should do in order to make communication go successfully and effectively. The concern of the present study is how to produce intentional ambiguity by means of violating CP and its maxims deliberately and why the addressor flouts the maxims. Horn (1984) reformulates Grice's in terms of an opposition between two principles: the Q-principle, which says that the speaker ought to provide as much information as possible, and the R-principle, which says that the speaker ought to be as economical as possible. The R-principle, namely the requirement that communication be as economical as possible, leads to an additional type of implicature. The Q-principle (Hearer-based) includes: (1) Make your contribution sufficient (Quantity). (2) say as much as you can (given R). The R-principle (Speaker-based) includes: (1) Make your contribution necessary (Relation, Quantity, Manner). (2)Say no more than you must (given Q). 2

The R-principle demands to avoid ambiguity, actually, people often violate the principle to produce ambiguous. For example, a couple has been married for a long time, but they have not got any children, when asked about it, the husband answers that:

She cannot bear children.

She cannot $g$ ive a birth to children.

She cannot tolerate the noise made by children.

The husband violates the maxim of manner by using an ambiguous word "bear". His answer is not direct, but people can draw the implicature from the ambiguity that it is wise not to get a clear reply. So the husband not only maintains conversation, but also avoids being embarrassed .1.2 IC-analysis

IC-analysis is one of the remarkable achievements of American descriptive linguists, especially Bloomfield. $\mathrm{Hu}$ Zhunagling says that constituent is a term used in structural sentence analysis for every unit, which is a part of a larger linguistic unit. In Oxford Concise Dictionary of Linguistics, the word constituency's explanation is: Relation, especially in syntax, between a unit which is part of a large unit and the whole of which it is part. Eg. The adjective phrase "very friendly" is a constituent of the noun phrase "very friendly people". The immediate constituents of a unit are the largest such parts. E.g. the immediate constituents of "meeting very friendly people" are "meeting" and "very friendly" people; those of this second constituent are in turn "very friendly" and "people". The analysis of sentences into constituents is immediate constituent analysis (or IC-analysis). Another example: The girl plays the game, S (A), the girl (B), play the game(C), each part is a constituent. Constituent can be joined together with other constituents to form larger units. If two constituents, in the case of the example above, B (the girl) and C (play the game), are joined to form a hieroglyphically higher constituent A ("S", here a sentence), then $B$ and $C$ are said to be the immediate constituent of A. The object of IC analysis is tried to catch the hierarchy of constituent relationships of a sentence structure.

For example: Flying planes can be dangerous. "Flying planes" can be understood as both a noun phrase and a nonfinite clause. When we look on it as a noun phrase, the internal semantic relation is that "flying" is the classifier and "planes" is the Head; when we look at it as a non-finite clause, flying planes" is an abstraction of Macro-thing.

Look at more examples:

IC-analysis not only states the presence of ambiguity, but also tells the number of ambiguous structures. And thus it is more effective than traditional grammar. 
IC-analysis is more complicated than traditional grammar which simply describes a sentence in linear order. IC-analysis represents a clearer and more thorough description of the internal constituent hierarchical structure of a sentence. Ambiguity is suggested, when IC-analysis segments a sentence in more than one way. But do ambiguous structures necessarily lead to ambiguous sentences? The answer is "No". Whether an ambiguous structure produces an ambiguous sentence is determined by the lexical s-selection.

\begin{tabular}{|l|l|}
\hline $\begin{array}{l}\text { (1) Patent medicines are sold by } \\
\text { frightening people. }\end{array}$ & $\begin{array}{l}\text { One interpretation is "frightening } \\
\text { people sell patent medicines", the } \\
\text { other "one way to sell patent } \\
\text { medicines is to frighten people." }\end{array}$ \\
\hline (2) We get down from the duck. & $\begin{array}{l}\text { The word "down" could be seen } \\
\text { as an adverbial, which means "from } \\
\text { the higher position to a lower." The } \\
\text { word "down" could also be seen as } \\
\text { a noun, which means of "the fluffy } \\
\text { feathers forming the first plumage } \\
\text { of a young bird. }\end{array}$ \\
\hline (3) In the film, Pat was a witch. & $\begin{array}{l}\text { This sentence is ambiguous, for } \\
\text { Pat can be either one character in } \\
\text { the film or one actor who acts as a } \\
\text { witch in the film. }\end{array}$ \\
\hline 3 & \multicolumn{1}{|c|}{$2 / 1$} \\
\hline
\end{tabular}

\section{Sound Ambiguity and Meaning Ambiguity}

As a linguistic phenomenon, ambiguity is universal and common, existing in all languages. Ambiguity nearly exists everywhere. According to different points of view, it can get different classifications. The author divides it into two levels: sound ambiguity and meaning ambiguity. The author will select some good examples to show the ambiguity in our daily life and literature, especially in positivity.

\subsection{Ambiguity Caused by Sound}

Generally speaking, sound ambiguity is mainly caused by polysemy, homonymy, variation of stress and tone, liaison, etc. Polysemy and homonymy are the main causes of sound ambiguity. Extension of meaning is the main source of polysemy and homonymy.

Polysemy is also called radiation or multiplication. This happens when a word gets a wider range of meanings. For example, "paper" comes from Greek papyrus.It (or polysemia) is a compound noun for a basic linguistic term. The name comes from Greek poly (many) and semy (to do with meaning, as in semantics). So the coexistence of many possible meanings for a word or phrase called polysemy. Polysemy is a term used in semantic analysis to refer to lexical item which has a range of different meanings. It is a basic feature of human speech, which can occur in a large number of ways and it often comes up through a kind of verbal shorthand. In every trade or profession, in every situation there is a certain thought which is so much present to one's mind. There is a class, called "complementary polysemy" wherein a single verb has multiple senses, which are related to one another in some expected way. An example is "bake", which can be interpreted as a change-of-state verb or as a creation verb in different circumstances. "John baked the potato." (change-of-state, verb) "John baked a cake." (creation, verb). An overwhelming majority of words are polysemous. The reason for polysemy is the lack of information. Sometimes even if there is a polysemy in an actual expression, there is no ambiguity. 
Here are more examples:

Gentleman, we must all hang together, or we shall most assuredly all hang separately.

In the example, the speaker deliberately uses ambiguity to achieve certain communicative needs. This is a famous statement made by Benjamin Franklin at the signing of the Declaration of Independence. The first "hang" means "to unit together", while the second "hang" means "kill (someone) by tying a rope attached from above around their neck and removing the support from beneath them (often used as a form of capital punishment)". Here Franklin intentionally publicizes his political stand.

A got married recently. B was one of A's friends. One day, B ran into A on the street.

B: How is your married life?

A: Marriage is not a word. Marriage is a sentence, a lifelong sentence.

"Sentence" is a polysemous word. The first interpretation is "a grammatical unit", and the second interpretation is "the punishment fixed by law for a particular offence". By deliberately making use of the word "sentence", the speaker describes his feeling towards his marriage in a self-mocking way.

Polysemy is a fundamental feature of human speech, which can arise in a multiplicity of ways. Some sources are native and some are under the influence of a foreign language. Compare with polysemy, homonymy is more common.

Homonymy is each of two or more words having the same spelling and pronunciation but different meanings and origins (e.g. ball for a spherical object and for a social function for dancing). It is a common phenomenon in both English and Chinese. Meanings of homogeneous words have no relationship with each other. Homophones will cause confusion in decision of accurate word sense. For an example, bear can be a noun describing an animal or a verb meaing to produce or to put up with somebody or something. In Alice's Adventures in Wonderland:

"Mine is a long and sad tale!'Said the Mouse, turning to Alice, and sighing.

"It is a long tale, certainly."Said Alice, looking down with wonder at the mouse's tail, "but why do you call it sad?"3

In the dialogue, tale and tail have the same pronunciation and both are modified by long. It sounds precise and ordinary, so Alice wrongly regarded "long tale" as "long tail".

"How is bread made?"

"I know that!"Alice cried eagerly, "You take some flour"

"Where do we pick flower?"the White Queen asked, "In a garden or in the hedges?"

"Well, it isn't picked at all,"Alice explained, "It is ground"

"How many acres of ground?" said the White Queen4.

The dialogue shows that the author has produced a pun through a play on a couple of homophones-flour and flower, in addition to a pair of complete homonyms-which also contains ambiguity. The author made good use of ambiguity to make dialogue humorous and lively.

When an expression is read with different intonation types or different placement of stress, it tends to be ambiguous since intonation and stress play important parts in meaning construction. For example:

(1) My young brother had a/greidei/.

We may interpret this utterance as: (a) My younger brother has a grade A. (b) My younger brother has a great day.

Another example is:

(2) Teacher: What are usually used as a conductor of electricity?

Student: Why- er?

Teacher: Correct, Wire. Now tell me, what is the unit of electricity power?

Student: What?

Teacher: That's absolutely right, the watt.

"Why-er" and "wire", "what" and "watt" has the pronunciation. This is a good example.

From examples above, people can see that ambiguities which cannot appear written form may arise in spoken language. This mainly caused by liaison, stress etc., among phrases or sentences. 
As is mentioned above, shifts in application will result in polysemy. The reason for polysemy is a lack of information. Sometimes even if there is a polysemy in an actual expression, there is no ambiguity. Among those shift, a major one is the shift of word scope. But homonymy is far less common and far less complex than polysemy, through its effect can be just as can be just serious and even more dramatic. Both polysemy and homonymy can arise in native ways or under the influence of foreign languages.

\section{Sound and Meaning Ambiguity in Use}

British linguist William Empson holds that ambiguity is the enemy people have to watch. Language cannot exist without ambiguity, which has represented both a curse and a blessing through the ages. If ambiguity is not avoided, it may make communication difficult. However, not all ambiguities are negative. Ambiguity also can create a special rhetorical effect or achieve a certain pragmatic goal.

\subsection{Negativity}

When people come across ambiguity during the course of studying English as a foreign language, we sometimes get into trouble just because we misunderstand the true meaning. Even in some situation, such a mistake may lead to a mess result. This indicates that it is a great need to resolve ambiguity. Efforts should be made into and attention should be paid to the resolution of ambiguity in linguistic research so as to achieve successful and effective communication. The overlapping meanings emerge from the tropes, ways of saying something by always saying something else. In this sense, ambiguity in literature has a very dark side, when important documents are interpreted in different ways, resulting in persecution, oppression, and death. English ambiguity is both common and complex fact, and usually has a negative effect to the inconvenience of our communication. People are in the process of spoken communication should be as much as possible to avoid ambiguity.

The major means to eliminate ambiguity is provide context. Context can not only cause ambiguity but also eliminate ambiguity. Context restriction means context selection and context interpretation. The author takes an ambiguous phrase as an example: He is a hard businessman. The word "hard" in this context can mean both "hardworking" and"difficult". The text fails to narrow down the meaning so that it is difficult for the reader to decide what exactly the speaker means. But if the background information can give a hint there would be no misunderstanding. For example, we know that he works more than ten hours in his company every day. It is clear that here "hard" means"hardworking".

Another example is: The fish is ready to eat 7.

a. The fish is cooked or served, so ready for people to eat.

b. The fish is ready to eat things.

On a grammatical basis, both sentences can have two different interpretations. But if we add some modification, we will achieve clarity:

What a nice smell! The fish is ready to eat.

Another means to eliminate ambiguity is to the replacement. For example "She didn't take my tip." "Tip" can be understood as "a sum of money given to someone as a way of rewarding them for their services". And another understanding is "a small but useful piece of practical advice". The word "tip" changes into the corresponding synonym. Just like "She did not take my money." or "She did not take my advice." And people can provide the context "She did not take my tip, so he lost a lot of money." or "she did not take my tip because she did that for nothing." the sentence will be clear. Another example: "A nice ball!" This Sentence is ambiguous, because "ball" is a homonym. It can refer to "a dancing party" or "solid or hollow sphere used in games". However, the context of "speaking right after a dancing party" can dispel the ambiguity easily and clearly.

Ambiguity is a fundamental problem of language comprehension and a problem of communication. So people should avoid ambiguity through above methods. Ambiguity should be actively excluded in order to avoid misunderstanding. 


\section{CONCLUSION}

The descriptive and analytical work of the paper is now complete. To conclude, the author will sketch out the train of thought of this paper to show her major findings and limitations, followed by a few implications for further work.

Language is a very complex phenomenon in which there are kinds of ambiguities. Since the discussion about linguistic ambiguity is not new and many language schools have done studies on the topic in the history, the author has reviewed the study on ambiguity from a history perspective. Basing on the Q-and R-principle and ICanalysis, the present research develops a conceptual framework with a view of supplying comprehensive and systematic explanation of sound ambiguity and meaning ambiguity. The R-principle demands to avoid ambiguity. Through IC-analysis, not only is ambiguity proved, but also is the number of the ambiguities in the process of analyzing ambiguity. Sound ambiguity includes polysemy, homonymy and variation of stress and tone, liaison, etc. Polysemy and homonymy are the main causes of sound ambiguity. Shifts in application will result in polysemy. The reason for polysemy is a lack of information. Homonyms frequently appear in communication, especially in advertisements and literary works. A competent addressor will use homonyms to create special communicative effects. As for meaning ambiguity, without disparity in meaning, there will not exist ambiguity. If people want to have a discussion about ambiguity, first of all, people have to make it clear what meaning is. Culture and context are the basic causes of meaning ambiguity. Culture is closely related with language. Context is the main issue that makes ambiguity arise.

The author's analysis supports the view that sound ambiguity and meaning ambiguity widely exist in our daily life. This study aims to comprehend the sound ambiguity and meaning ambiguity, and understand their relationship. It should be pointed out that in authentic language sound and meaning ambiguity do not always appear separately; instead they might mingle together and reside in one sentence at the same time. Everything has two aspects. Ambiguity has positive and negative function: sometimes ambiguity give people fun in the life. Ambiguity plays an important part in creating humorous effects, expressing two meanings in a pun, irony and euphemism. The author also points out its functions of politeness-achieving and self-protecting. As to what kind of effects it can achieve, people have noticed and studied its rhetorical functions for a long time.

\section{References}

[1] A S Hornby, Sally Wehmeier. Oxford Advanced Learner's English and Chinese Dictionary [M]. Beijing: Oxford University Press\& The Commercial Press, 2004.

[2] Jack C.Richards, Richard Schmidt, Heidi Kendrick, Youngkyu Kim. Longman Dictionary of Language Teaching and Applied Linguistics [M]. Beijing: Foreign Language Teaching and Research Press, 2005.

[3] Qi Hui. Study on Pragmatic Functions of Ambiguity [MA]. Southeast University, 2006.03.

[4] R.H.Robins. A Short History of Linguistics [M]. Beijing: Foreign Language Teaching and Research Press, 2001.

[5] Rao Shuangyan. A Pragmatic Study of Intentional Ambiguity [MA]. Shanghai International Studies University, 2008.12. 